\title{
Density functional study of the electronic and vibrational properties of $\mathrm{TiOCl}$
}

\author{
L. Pisani ${ }^{1}$, R. Valentí ${ }^{2}$, B. Montanari ${ }^{3}$, and N. M. Harrison ${ }^{1,4}$ \\ ${ }^{1}$ Department of Chemistry, Imperial College London, \\ South Kensington campus, London SW7 2AZ, United Kingdom \\ ${ }^{2}$ Institut für Theoretische Physik, Universität Frankfurt, 60438 Frankfurt, Germany \\ ${ }^{3}$ STFC Rutherford Appleton Laboratory, Chilton, \\ Didcot, Oxfordshire OX11 OQX, United Kingdom \\ ${ }^{4}$ STFC Daresbury Laboratory, Daresbury, Warrington WA4 4AD, United Kingdom
}

(Dated: October 27, 2018)

\begin{abstract}
We present the phonon spectrum of $\mathrm{TiOCl}$ computed using hybrid density functional theory (DFT). A complete analysis of the spectrum is performed for the space group Pmmn (high symmetry phase) and the space group $P 2_{1} / m$ (low symmetry phase), which is the symmetry of the spin-Peierls phase. We show that the nonlocal correlations present in the hybrid DFT approach are important for understanding the electronlattice interactions in $\mathrm{TiOCl}$. The computed frequencies compare well with those observed in Raman and infrared spectroscopy experiments and we identify the origin of an anomalous phonon observed in Raman spectroscopy. The relationship between relevant zone boundary phonons in the high symmetry phase and the zone center counterparts in the $P 2_{1} / m$ symmetry allow us to speculate about the origin of the spin-Peierls phonon.

PACS numbers: 71.15.Mb,63.20.-e,78.30.-j
\end{abstract}

\section{INTRODUCTION}

The unconventional properties of the low-dimensional spin-1/2 compound $\mathrm{TiOCl}$ have been a subject of intensive debate in recent years $1,2,3,4,5,6,7,8,9,10,11,12,13,14$. This system shows two consecutive phase transitions at $T_{c_{2}}=91 \mathrm{~K}$ and at $T_{c_{1}}=60 \mathrm{~K}$ from a magnetic lowdimensional behavior to a spin-Peierls dimerized state ${ }^{1.4}$ through an intermediate, structurally incommensurate, phase. While the conventional spin-Peierls phase in a spin chain is well understood in terms of the magnetoelastic coupling, the behavior in $\mathrm{TiOCl}$ is more complicated. The $a b$-planes of $\mathrm{TiOCl}$ consist of $\mathrm{TiO}$ bilayers separated by layers of $\mathrm{Cl}^{-}$ions stacked along the $c$ direction ${ }^{1}$. This bilayered structure, in conjunction with the localized nature of the Ti $3 d$ electrons, has motivated various proposals about the role of interchain frustration ${ }^{5}$ (within one layer and between layers), lattice degrees of freedom and correlation effects $\frac{6,7.10,12}{1}$ at the phase transitions. Initial suggestions about the influence of orbital fluctuations ${ }^{2}$ have been settled both experimentally and theoretically and the groundstate of the system at temperatures above $T_{c_{2}}$ is now known to be characterised by singly occupied Ti $3 d_{x y}$ orbitals $4,5,6,10$.

The ground state electronic structure of this system in the observed room temperature structures has been calculated within $a b$ initio density functional theory $(\mathrm{DFT})^{1,3,4,6}$. The role of electronic correlations beyond those included in the local density approximation (LDA) have also been studied using the $\mathrm{LDA}+\mathrm{U}^{1,3,6}$, $\mathrm{LDA}+\mathrm{DMFT}{ }^{10,11}$, and very recently the LDA+cluster$\mathrm{DMFT}^{12}$ approximations. In the latter approach, where two-site correlations were considered, a good agreement between the calculated spectral function and the measured photoemission and $\mathrm{O}$ K-edge X-ray absorption spectra was achieved; for all other methods mentioned the computed band gap is significantly below that observed. These studies provide strong evidence for the important role played by electron correlations in this material.

The interplay between the structural and electronic degrees of freedom within the various phases, which is particularly relevant in the study of the anomalous spinPeierls transition, has only been partially investigated from a theoretical point of view. In a previous study by some of the authors ${ }^{7}$, the Raman active $A_{g}$ phonon modes in TiOCl for the high-symmetry (HS) phase $(T>$ $T_{c_{2}}$ ) were obtained within an $\mathrm{LDA}+\mathrm{U}$ formalism and the frozen-phonon approach. It was concluded that the inclusion of electron correlation at the $\mathrm{LDA}+\mathrm{U}$ level significantly improved the agreement between the computed and observed frequencies. In the present work we extend these studies to include the $P 2_{1} / m$ phase, which has been identified by Palatinus et al $\stackrel{15}{\underline{15}}$ as the low-symmetry (LS) phase $\left(T<T_{c_{1}}\right)$. We present the electronic structure and a complete classification of the phonon spectrum for both the HS and the LS structures, and make a detailed comparison with the measured Raman and infra-red spectra. We also extend the treatment of electron correlation to the hybrid exchange DFT approximation - which includes both the local (U) and non-local components of exchange and correlation beyond those present in the LDA. The much better agreement with the observed spectra compared to that achieved in previous calculations (within the LDA and LDA+U $)^{7}$ strongly suggests that nonlocal correlations are important to describe the effects of lattice vibrations in TiOCl. Within this theoretical framework we can also identify for the first time the anomalous phonon observed in Raman spectroscopy ${ }^{2}$ which appears to violate the Raman selection rules. Moreover, 
by analysing the relationship between the zone boundary phonons in the $b$ direction (Ti-chain axis) in the HS phase and relevant zone center phonons in the $P 2_{1} / m$ phase, we suggest possible candidates for the spin-Peierls phonon mode.

The paper is organized as follows: in section II we present the computational details of our calculations, in section III we discuss the electronic structure of $\mathrm{TiOCl}$ in the HS phase within the various approximations used in previous studies and present our results using hybrid exchange DFT. Sections IV and V report on the optimization of the lattice parameters and the calculation of phonons for the HS phase. In section VI we compute and discuss the phonons in the $P 2_{1} / m$ symmetry (LS phase) and in the last section we summarize our conclusions.

\section{COMPUTATIONAL DETAILS}

The first-principles calculations presented here have been performed using the hybrid exchange density functional B3LYP 16.17 as implemented in the CRYSTAL package $\frac{18}{}$. In CRYSTAL, the crystalline wave functions are expanded as a linear combination of atom centered Gaussian orbitals LCAO with $s, p, d$, or $f$ symmetry. The $\mathrm{Ti}, \mathrm{O}$ and $\mathrm{Cl}$ atoms are described by a triple valence all-electron basis sets: an $86-411 \mathrm{G}^{* *}$ contraction (one $s$, four $s p$, and two $d$ shells), an $8-411 \mathrm{G}^{*}$ contraction (one $s$, three $s p$, and one $d$ shells) and an 86-311G* (one $s$, four $s p$, and one $d$ shells), respectively. A reciprocal space sampling on a Monkhorst-Pack grid 19 of shrinking factor equal to 6 is adopted after finding it to be sufficient to converge the total energy to within $10^{-6} \mathrm{eV}$ per unit cell. The Gaussian overlap criteria which controls the truncation of the Coulomb and exchange series in direct space are set to $10^{-7}, 10^{-7}, 10^{-7}, 10^{-7}$, and $10^{-14}$ the details of which are presented elsewhere $\frac{18}{8}$. Typically linear mixing of $70 \%$ and an Anderson second-order mixing scheme was used to guide the convergence of the self-consistent field procedure.

The geometry optimizations are performed using the algorithm proposed by Schlegel et al .20 . The full dynamical matrix was computed using finite differences of the analytic gradients for which the atoms were displaced by $0.02 \AA$.

\section{ELECTRONIC STRUCTURE}

In Ref. 7, the authors performed an investigation of the electronic structure of $\mathrm{TiOCl}$ at the experimental cell parameters of the HS phase (space group 59 Pmmn) using the LDA and LDA $+\mathrm{U}$ methods. It was concluded that the LDA functional does not reproduce the measured electronic structure or the internal coordinates of the atoms in the cell, whereas within the $\mathrm{LDA}+\mathrm{U}$ method the Mott-Hubbard insulator nature of TiOCl is obtained as well as the internal coordinates. Neverthe- less, the LDA $+\mathrm{U}$ approximation does not describe properly the optical band-gap of $2 \mathrm{eV}$ observed in infrared spectroscopy experiments. While the gap between the occupied spin up $d_{x y}$ band and the unoccupied spin down $d_{x y}$ is computed to be $2.8 \mathrm{eV}$ within the $\mathrm{LDA}+\mathrm{U}$ model using $\mathrm{U}$ and $\mathrm{J}$ values of $3.3 \mathrm{eV}$ and $1 \mathrm{eV}$ respectively, the gap between the occupied $d_{x y}$ and unoccupied $d_{x z-y z}$ of the same spin is only $0.3 \mathrm{eV}$. Since the optical gap is a measure of the lowest energy difference between occupied and unoccupied electronic states, the LDA+U calculations significantly underestimate the measured value.

Saha-Dasgupta et al. improved on the description of on-site correlation by using a single-site LDA+DMFT, where dynamical fluctuations beyond the $\mathrm{LDA}+\mathrm{U}$ are introduced using a quantum monte carlo impurity solver $\underline{10}$. This method provides a better description of the overall width of the spectral function, but the calculated band gap of $0.5 \mathrm{eV}$ still underestimates very significantly the observed gap. LDA+DMFT calculations performed with an iterated perturbation theory (ITP) impurity solver 11 yield a bigger energy gap at the cost of introducing a mixing of the $d$-orbitals significantly greater than that deduced from the spectroscopy ${ }^{5.6}$.

Based on the fact that $\mathrm{TiOCl}$ is a low-dimensional correlated system and that at low temperature it dimerizes into Ti-Ti singlet pairs along the $b$-axis, it has been suggested 12 that two-site correlations may be important even at high temperatures and thus that the single site approximation will be insufficient. We note that in Raman spectroscopy ${ }^{2}$, specific heat ${ }^{\frac{9}{}}$ and NMR experiments ${ }^{21}$ strong evidence is found for the existence of low energy fluctuations of the spin system in a temperature range that extends well above the spin-Peierls transition. The role of inter-site correlation has been investigated previously in Ref. 12 by using the cluster extension of DMFT in which the correlated impurity is taken to be Ti-Ti pairs instead of a single site $\mathrm{10}^{0}$. The computed linewidth, the lineshape and the band gap of the calculated spectral function are found to be in good agreement with angle integrated photoemission data and with O K-edge absorption spectra 22 .

In the following, we start from a completely different approach and demonstrate that the role of nonlocal correlations can be also captured in an effective manner within the hybrid DFT approach.

The LDA+U scheme is known to lack any description of non-local (off-site) electron exchange and correlation beyond the local density approximation (LDA) due to the fact that the empirical Hubbard U term added to the Hamiltonian improves only on the description of on-site Coulomb effects. In order to capture the non-local component of the electronic exchange and correlation (which is unsatisfactorily approximated within the LDA) one would need to extend the on-site $\mathrm{LDA}+\mathrm{U}$ treatment to inter-site interactions, which therefore would take the form of an extended version of the $\mathrm{LDA}+\mathrm{U}$ method, namely the $\mathrm{LDA}+\mathrm{U}+\mathrm{V}$ scheme. Within this scheme, the inter-site term $\mathrm{V}$ is treated within the Hartree-Fock 
(mean-field) approximation (as the $\mathrm{U}$ term is in $\mathrm{LDA}+\mathrm{U}$ ) and thus the scheme is sufficient to introduce the $\mathrm{Ti}-\mathrm{Ti}$ pair interaction neglected in the LDA. In hybrid DFT, the treatment of off-site exchange and correlation effects beyond LDA is already built in due to the inclusion in the Hamiltonian of a Fock-exchange term which operates between all (on-site and off-site) Kohn-Sham orbitals. The effectiveness of this term for describing the on-site correlation is well documented ${ }^{22}$ and there is every reason to believe that it will be equally effective in describing the non-local exchange and correlation effects beyond LDA at a mean-field level.

Fig. 1 shows the spin polarized band structure of Ti$\mathrm{OCl}$ calculated within the B3LYP approximation for the experimental HS structure ${ }^{8}$. We note that the band structure does not change significantly when calculated at the relaxed HS geometry. We observe that, in contrast with the $\mathrm{LDA}+\mathrm{U}$ approach, the adoption of the B3LYP approximation improves significantly on the description of the electronic ground state by opening a band gap of about $2.2 \mathrm{eV}$, in good agreement with the measured gap. The band states just below the Fermi level are majority spin states of $d_{x y}$ character (Fig. 11 left panel). The coordinate system has been chosen with $z$ in the same direction as $a$, and with the $x$ and $y$ axes rotated by $45^{\circ}$ with respect to $b$ and $c$. Above the Fermi level on the majority spin channel (Fig. 1, left panel) we find, in order of increasing energy, band states with $d_{x z-y z}, d_{x z+y z}, d_{x^{2}-y^{2}}, d_{z^{2}}$ character and likewise in the minority spin panel (Fig. 11 right panel), with the only difference that the $d_{x y}$ spin down states are unoccupied and placed between the $d_{x z-y z}$ and the $d_{x z+y z}$ states. It is worth pointing out that the bands in Fig. 11 have been computed for a ferromagnetic alignment of $\mathrm{Ti}$ spins. In the antiferromagnetic case (not shown) the band structure of up and down states is symmetric, as in any conventional antiferromagnet, and the band gap is found to be the same as in the ferromagnetic case. The states at the boundary of the band gap are of $d_{x y}$ symmetry (occupied) and of $d_{x z-y z}$ symmetry (unoccupied).

The above features on the orbital nature of the states around the band gap are in full agreement with previous results obtained within $\mathrm{LDA}+\mathrm{U}^{1,3}$.

\section{LATTICE STRUCTURE}

In Table \ we present the equilibrium lattice parameters of the HS phase obtained after a full relaxation of the atomic structure of the FM state within the B3LYP approximation. For comparison, we also show the results obtained with the gradient corrected functional of Perdew, Burke and Ernzerhof ${ }^{23}$ (PBE) and the experimental values 8 .

While the agreement between the calculated and measured $a$ parameter is reasonably good, this is not the

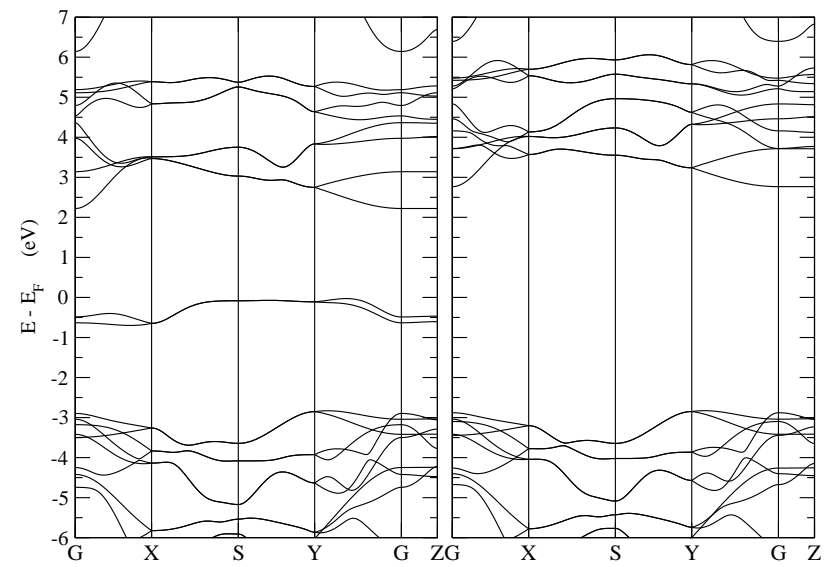

FIG. 1: Calculated band structure for the high symmetry phase of $\mathrm{TiOCl}$ at the experimental crystal structure along the path $\Gamma-\mathrm{X}-\mathrm{S}-\mathrm{Y}-\Gamma-\mathrm{Z} . \quad(\Gamma[0,0,0] ; \mathrm{X}[1 / 2,0,0] ; \mathrm{S}[1 / 2,1 / 2,0]$; $\mathrm{Y}[0,1.2,0] ; \mathrm{Z}[0,0,1]$ ) The majority and minority spin bands are shown in the left and right panel, respectively. $E_{F}$ indicates the Fermi energy.

case for $c$ and $b$. The strong disagreement in the case of the $c$ parameter is due to the predominantly van der Waals type of binding between bilayers in the $c$ direction which is not correctly described in LDA, GGA or B3LYP approximations to DFT. Keeping $c$ fixed at the experimental value and fully optimising the structure does not change significantly the values of the $a$ and $b$ parameters. We notice that the energy landscape is rather flat when varying the length of the $c$ lattice constant due to the very weak binding. However, we find no significant effect of the $c$ parameter on the assignment of the phonon modes in the Raman and infrared spectra in the range of frequencies considered in the next section.

Concerning the parameter $b$ (chain direction), the B3LYP functional gives an overestimation of $b$ of about $6 \%$. In order to understand this issue, we employed first the PBE functional for the full structural optimisation of the HS phase and then compared with B3LYP results. With the choice of the PBE functional, we find that the structure is unstable with respect to Ti interchain dimerisation, where neighbouring chains shift rigidly towards each other, leaving the intra-chain step unchanged and producing an alternating interchain Ti-Ti distance. This is due to the fact that the electronic structure is found to be a fully spin polarised metallic state, due to the self-interaction error (SIE) present in the GGA. The orbital polarisation found in SIE compensated functionals $(\mathrm{LDA}+\mathrm{U}$ and B3LYP) is lost within PBE and the orbital $d_{x y}$ becomes partially unoccupied in favour of the $d_{x z-y z}$ orbital. The latter points towards Ti atoms of neighbouring chains and therefore favours interchain dimerisation.

This observation is useful in interpreting the behaviour of the B3LYP hybrid functional. We note here that the functional of Lee, Yang and Parr 24 (LYP) that describes the exchange-correlation term in B3LYP, belongs to the family of the PBE functional, and thus we expect it to 
provide similar results as PBE. However, the inclusion of Fock exchange removes the interchain instability found within the PBE functional and enables the B3LYP approximation to describe reasonably well the geometric and electronic structure simultaneously.

By changing the amount of Fock exchange from the amount prescribed for the B3LYP functional, we are able to conclude that the overestimation of $b$ is strongly related to the percentage of the Fock exchange term in the Hamiltonian. Possible explanations of the disagreement between the experimental and theoretical values of $b$ are the following: (i) the exchange contribution in B3LYP is overestimated due to the neglect of screening leading to an overestimation of the local Pauli pressure and thus a stretching of $b$. (ii) more speculatively, if dynamical spin singlet fluctuations are important, they could bring the Ti atoms into a precursor dimerised state with no long-ranged crystallographic order. As a result, the x-ray measurement would probe a thermally averaged value of the $\mathrm{Ti}-\mathrm{Ti}$ distance $(=\mathrm{b} / 2)$ that is shorter than the ground state value. It is not clear at present which of these explanations accounts for the overestimation of the $b$ parameter.

In Table II we show the relevant equilibrium atomic distances and angles. In general a very good agreement of B3LYP results with the experimental values is obtained.

TABLE I: Equilibrium lattice parameters of the high symmetry unit cell.

\begin{tabular}{|c|c|c|c|}
\hline & $\mathrm{a}(\AA)$ & $\mathrm{b}(\AA)$ & $c(\AA)$ \\
\hline exp. & 3.78 & 3.34 & 8.03 \\
\hline PBE & 3.98 & 3.31 & 8.57 \\
\hline B3LYP & 3.81 & 3.49 & 8.69 \\
\hline
\end{tabular}

TABLE II: Equilibrium atomic distances and angles in the high symmetry unit cell.

\begin{tabular}{ccccc}
\hline \hline & $\mathrm{Ti}-\left.\mathrm{O}\right|_{a}(\AA)$ & $\mathrm{Ti}-\left.\mathrm{O}\right|_{b}(\AA)$ & $\mathrm{Ti}-\mathrm{Cl}(\AA)$ & $\mathrm{Ti}-\mathrm{O}-\left.\mathrm{Ti}\right|_{a}$ \\
\hline exp. & 1.97 & 2.18 & 2.41 & $147.7^{\circ}$ \\
PBE & 2.02 & 2.13 & 2.43 & $160.9^{\circ}$ \\
B3LYP & 1.98 & 2.22 & 2.45 & $148.6^{\circ}$ \\
\hline
\end{tabular}

\section{PHONONS IN THE PACE GROUP $P m m n$}

Within space group 59 (Pmmn), identified as the high symmetry phase of $\mathrm{TiOCl}$, the $\Gamma$ point phonons are classified according to symmetry as follows:

$$
\Gamma=3 A_{g}+3 B_{2 g}+3 B_{3 g}+2 B_{1 u}+2 B_{2 u}+2 B_{3 u}
$$

Each mode involves atomic displacements along a single axis.
TABLE III: Phonon frequencies in the high symmetry phase of TiOCl. The first and second column denote respectively the irreducible representation and the multiplicity of the modes. The third column indicates the direction of the phonon displacements. The phonon frequencies are given in the fourth 3 -set columns and we include the reported experimentally observed frequencies in the fifth 3 -set columns.

\begin{tabular}{|c|c|c|c|c|}
\hline \multicolumn{5}{|c|}{ RAMAN } \\
\hline Ir.Rep. & Mult. & Displ. & Freq. & (experim.) \\
\hline$A_{g}$ & 3 & $c$ & 205342443 & $(203)(365) \quad(430)$ \\
\hline$B_{2 g}$ & 3 & $a$ & 116256613 & \\
\hline$B_{3 g}$ & 3 & $b$ & $\begin{array}{lll}141 & 287 & 431\end{array}$ & \\
\hline
\end{tabular}

\section{INFRARED}

\begin{tabular}{cccc|c}
\hline \hline \multicolumn{2}{c}{ Ir.Rep. Mult. Displ. } & \multicolumn{2}{c}{ Freq. } & (experim.) \\
\hline$B_{1 u}$ & 3 & $c$ & -328514 & \\
$B_{2 u}$ & 3 & $b$ & -256274 & $-(177)(294)$ \\
$B_{3 u}$ & 3 & $a$ & -154463 & $-(104)(438)$ \\
\hline
\end{tabular}

The modes with symmetry $A_{g}, B_{2 g}$, and $B_{3 g}$ are along the $c, a$ and $b$ axis, respectively, and are Raman active. The modes with symmetry $B_{1 u}, B_{2 u}$, and $B_{3 u}$ are along the $c, b$ and $a$ axis, respectively, and composed of one acoustic and two optic modes, the latter being infrared active.

In Table III the calculated frequencies are reported and classified according to direction of displacement and irreducible representation.

The frequencies of the $A_{g}$ modes at 205, 342 and 443 $\mathrm{cm}^{-1}$ compare quite well with the experimental values 2 as do the values computed within the $\mathrm{LDA}+\mathrm{U}$ method in Ref. 7. No cross polarisation of light was considered in Ref. 2 and therefore no experimental values for $B_{2 g}$ and $B_{3 g}$ are available.

Comparison with infrared measurements ${ }^{25}$ for the $B_{2 u}$ and $B_{3 u}$ modes, is quite satisfactory for the high frequency modes but less so for the low frequency ones. As a possible explanation for the discrepancy, we estimated the anharmonic corrections to the low frequency modes by examining the energy surface along the mode and found it to be harmonic to an excellent approximation. However, it is worth mentioning that the infrared active modes have been measured separately by Grueninger et $a l^{26}$ and a value of $201 \mathrm{~cm}^{-1}$ was found for the lower frequency $B_{2 u}$ mode, which agrees significantly better with the calculated frequency.

\section{PHONONS IN THE SPACE GROUP $P 2_{1} / m$}

\section{A. Phonon spectrum}

Within space group $11\left(P 2_{1} / m\right)$, which has been identified by Palatinus et al $\stackrel{15}{\underline{15}}$ as the spin-Peierls (LS) sym- 
TABLE IV: Phonon frequencies in the low symmetry phase. The first four columns have the same assignment as in Table III In the fifth column we include, where appropriate, the HS irreducible representation counterpart.

\begin{tabular}{|c|c|c|c|c|c|}
\hline \multicolumn{6}{|c|}{ RAMAN } \\
\hline Ir.Rep. & Mult. & Displ. & & Freq. & SG59 \\
\hline \multirow[t]{3}{*}{$A_{g}$} & 3 & $c$ & 203342 & 443 & $A_{g}$ \\
\hline & 3 & $b$ & 140289 & 431 & $B_{3 g}$ \\
\hline & 6 & $b c$ & 175212 & $285 \quad 323 \quad 382 \quad 490$ & \\
\hline \multirow[t]{2}{*}{$B_{g}$} & 3 & $a$ & 118257 & 616 & $B_{2 g}$ \\
\hline & 3 & $a$ & 122199 & 540 & \\
\hline
\end{tabular}

INFRARED

\begin{tabular}{|c|c|c|c|c|c|}
\hline$\overline{\text { Ir.Rep }}$ & Mult. & Displ. & & Freq. & SG59 \\
\hline \multirow[t]{3}{*}{$B_{u}$} & 3 & $c$ & -329 & 516 & $B_{1 u}$ \\
\hline & 3 & $b$ & -257 & 276 & $B_{2 u}$ \\
\hline & 6 & $b c$ & 175212 & 285323382490 & \\
\hline \multirow[t]{2}{*}{$A_{u}$} & 3 & $a$ & -159 & 464 & $B_{3 u}$ \\
\hline & 3 & $a$ & 122199 & 540 & \\
\hline
\end{tabular}

metry of $\mathrm{TiOCl}$, the $\Gamma$ phonons are classified as follows:

$$
\Gamma=12 A_{g}+10 B_{u}+6 B_{g}+5 A_{u}
$$

where one $A_{u}$ and two $B_{u}$ modes are acoustic.

In this space group, the $\mathrm{A}_{g}$ and $\mathrm{B}_{g}$ modes are Raman active while the $\mathrm{A}_{u}$ and $\mathrm{B}_{u}$ modes are infrared active. As a general feature, modes in this LS phase are either displacements within the $b c$ plane or purely along the $a$ axis. In particular, the $\mathrm{A}_{u}$ and $\mathrm{B}_{g}$ modes are along the $a$ axis and the $\mathrm{A}_{g}$ and $\mathrm{B}_{u}$ are within the $b c$ plane.

We note that starting from the experimental LS structure $\frac{15}{}$ within the space group 11 and fully optimising the structure results in a structure very close to the HS one with a unit cell doubled along the $b$-axis. This implies that the optimised structure does not show any signature of a spin-Peierls distortion. This is due to the fact that in the spin-Peierls mechanism the loss of elastic energy is overcompensated by an energy gain in spin-singlet fluctuations. The latter are not properly described within the current formulation of spin-DFT and therefore no magnetoelastic structural distortion can be expected for any of the exchange-correlation approximations currently in use. A consequence of this is that our zone center phonon mode calculations for the LS phase provide a good description of some relevant zone boundary phonons of the high symmetry phase, namely those phonons that are associated with a doubling of the HS unit cell along the $b$ axis and which are directly related to the spin-Peierls instability.

In Table IV the calculated frequencies and the directions of the corresponding displacement eigenvectors (within the reference system $a, b, c$ of the orthorhombic unit cell) are shown. Due to the absence of distortional features in the structurally optimised LS structure, the
18 modes of the HS phase are seen to evolve unchanged into the LS phase and the additional 18 modes, originating from the doubling of the unit cell, have degenerate features which will be discussed below.

In Table [V] we choose to decompose the LS modes according to their HS irreducible representation counterparts, where meaningful. It is important to note, however, that the spin-Peierls distortion does not modify the Raman active $\mathrm{A}_{g}$ modes' frequencies of the HS phase which are seen to evolve unchanged in the LS Raman spectra $^{2}$. We can therefore assume that the effect of lattice distortion is not crucial as far as the frequency calculation is concerned.

A peculiar feature of the two panels of Table IV is that the strictly LS Raman active modes are degenerate with their infrared counterparts i.e. the six $\mathrm{A}_{g}$ with the six $\mathrm{B}_{u}$ modes (third row in the upper and lower panel of Table IV) and the three $\mathrm{B}_{g}$ with the three $\mathrm{A}_{u}$ modes (fifth row in the upper and lower panel of Table [V]). The six $\mathrm{A}_{g}$ and six $\mathrm{B}_{u}$ modes are illustrated in Fig. 2 in order of increasing frequency value.

The degenerate character of these modes is due to the perfect triangular geometry of bonds between $\mathrm{Ti}$ atoms belonging to neighbouring chains that run along the $b$ axis. This geometry makes the interchain dimerisation patterns shown in Fig. 3 degenerate (this feature should be observed in some zone boundary phonons of the HS phase). In fact, if we were to consider the case of laddertype arrangement of chains, every Ti atom would have only one nearest neighbour in the neighbouring chain, and the two dimerisations shown in Fig. 3 would involve different lattice strains and therefore would be non degenerate. In the present case, where one chain is rigidly shifted by half of the lattice parameter $b$ with respect to the other, every $\mathrm{Ti}$ acquires two equivalent nearest neighbours along $a$ and therefore the in-phase and outof-phase displacements shown in Fig. 3 become equivalent. Note that this degeneracy would be split within a model which incorporates spin-singlet fluctuations, as pointed out above, although not significantly. However, in the non-dimerised phase $\left(\mathrm{T}>\mathrm{T}_{c 1}\right)$ such a degeneracy is restored.

All the six $\mathrm{A}_{g}$ and six $\mathrm{B}_{u}$ modes which originate in the LS phase represent possible dimerizations of the $\mathrm{Ti}$ chains within the $b c$ plane. The two lowest energy modes (at 175 and at $212 \mathrm{~cm}^{-1}$ ) keep the Ti-O distance along $a$ (see Table III) close to its optimised value, while the Ti$\mathrm{O}$ distance along $b$ oscillates, in a bond bending fashion for the mode at $175 \mathrm{~cm}^{-1}$ and as bond stretching mode for the one at $212 \mathrm{~cm}^{-1}$. In fact, the elastic constant associated with the Ti-O bond along $a$ is larger than the one along $b$ due to the shorter bond length. The Ti-O $\left.\right|_{a}$ bond sets the interchain elastic coupling and, due to its presence, the LS modes in the $b c$ plane are not merely displacements along the $b$ and $c$ axes independently, as one would expect if the Ti chains were not coupled. The higher frequency modes have larger $\mathrm{O}$ components and do not generally preserve the $\mathrm{Ti}-\left.\mathrm{O}\right|_{a}$ equilibrium distance, 
$175 \mathrm{~cm}^{-1}$

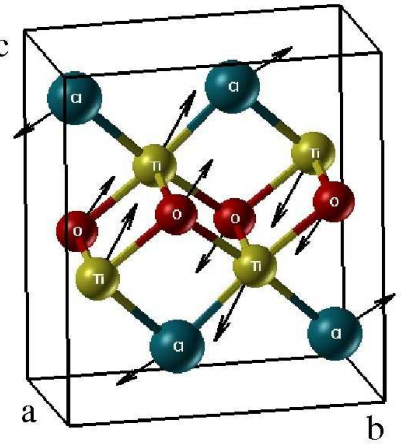

$\mathrm{B}_{u}$

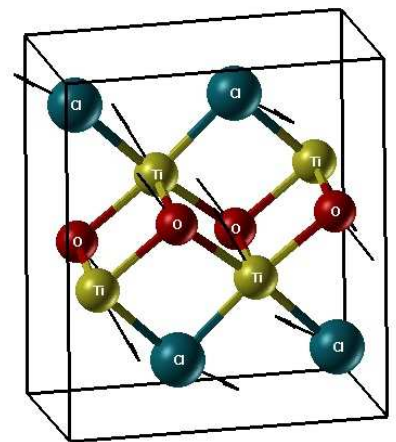

$212 \mathrm{~cm}^{-1}$
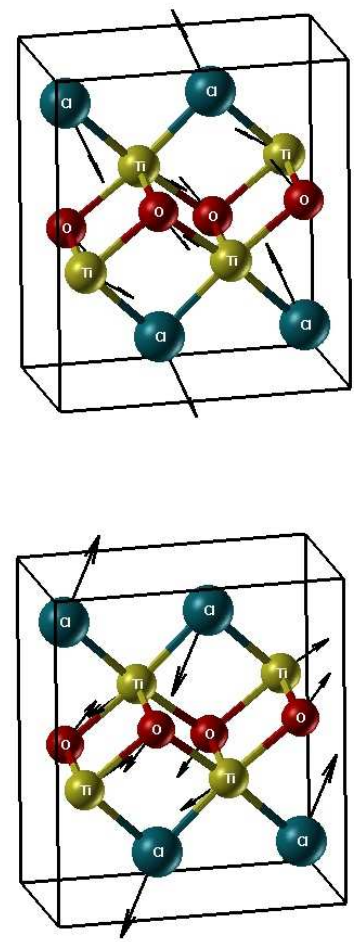

$285 \mathrm{~cm}^{-1}$
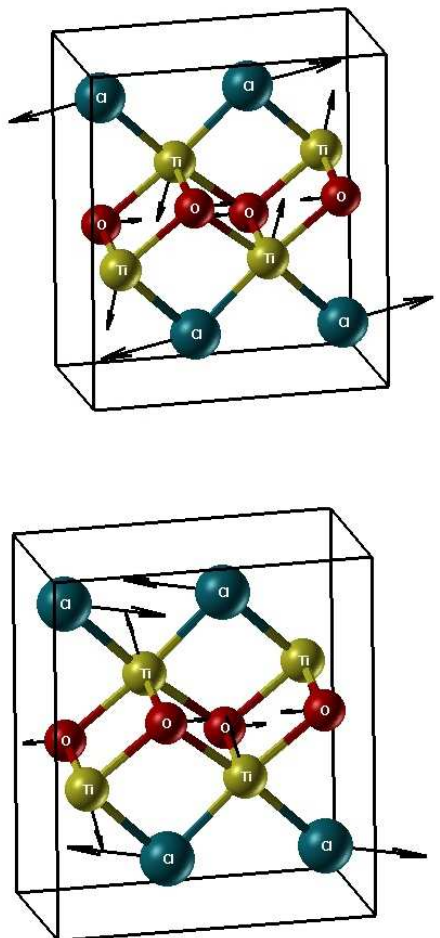
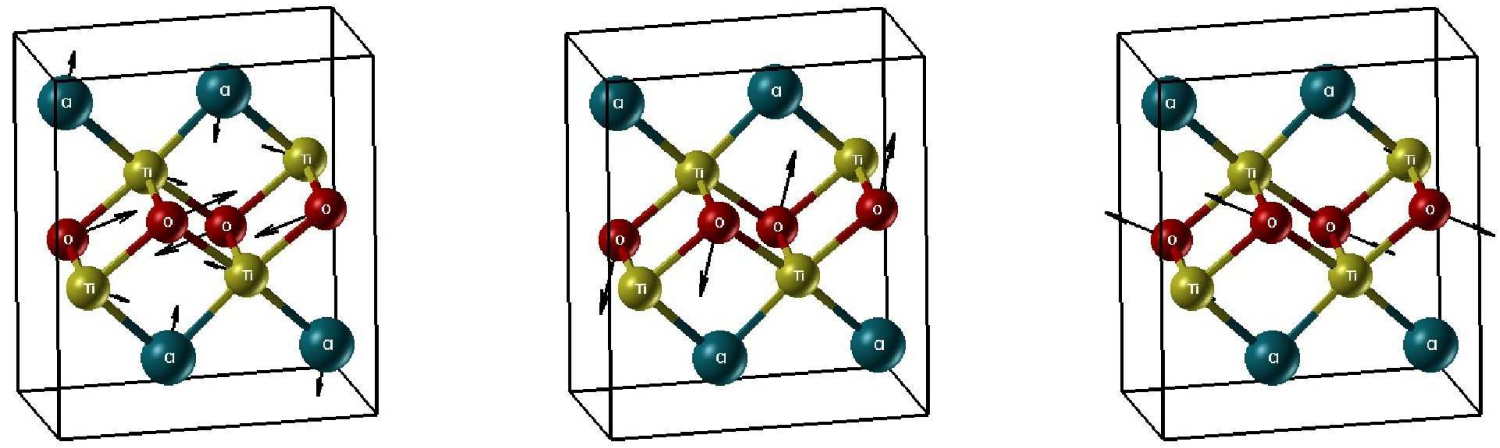

$382 \mathrm{~cm}^{-1}$

$490 \mathrm{~cm}^{-1}$

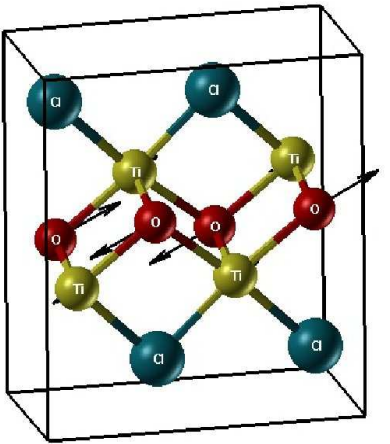

FIG. 2: (Colour online) Eigenvectors of the six $\mathrm{A}_{g}$ and six $\mathrm{B}_{u}$ modes originating in the low symmetry phase, grouped according to their degeneracy. Yellow spheres are Ti atoms, red oxygen and blue chlorine. The system of reference is indicated for the first mode on the top-left pannel and remains unchanged for the remaining modes. Length and direction of the arrows are directly proportional to the strength and vibrational direction of the modes. 


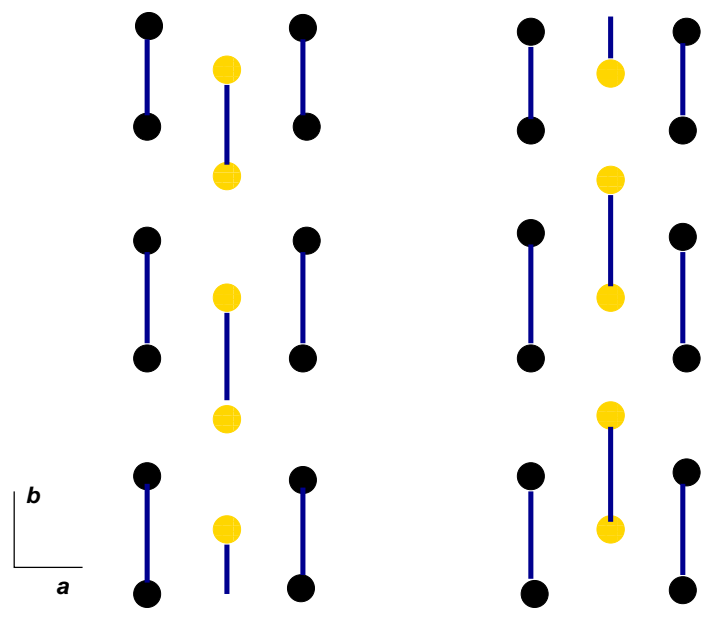

FIG. 3: (Colour online) Schematic representation of the two possible $\mathrm{Ti}-\mathrm{Ti}$ bond patterns within the $P 2_{1} / m$ symmetry group for TiOCl. Black and yellow symbols denote the $\mathrm{Ti}$ atoms on two consecutive layers of a single bilayer.

as inferable from the length and direction of the arrows representing the eigenvectors' components in Fig 2.

As a general comment on the six $\mathrm{A}_{g}$ and six $\mathrm{B}_{u}$ LS modes, it is interesting to note that they show a low dimensional character. They have a two-dimensional configurational space ( $b c$ plane) and due to the strong interchain coupling some of them show an effective onedimensional oscillation. This can be seen, for example, for the modes at 175 and at $212 \mathrm{~cm}^{-1}$ in Fig. 2 where the arrows on the $\mathrm{Ti}$ and $\mathrm{O}$ atoms have same length and direction, suggesting that the Ti-O-Ti-O plaquettes may be considered as an effective single vibrational block. The two modes represent the bending and the stretching of the bond between two neighbouring plaquettes. These features evidence the possible role of lowdimensional structural fluctuations as we shall discuss in subsection VIC,

\section{B. Phonon assignment and Raman spectra anomalies}

We note that the doubly degenerate lowest mode $\left(A_{g}\right.$, $\left.B_{u}\right)$ at $175 \mathrm{~cm}^{-1}$. i.e., the zone center phonon of the LS phase and a zone boundary phonon of the HS phase, (Fig. 2 first column, first and second raw panels) remarkably resemble atom displacements along the spin-Peierls distortion reported in Ref. 8 and Ref. 27 (compare Fig. 1(b) of Ref. 8 and Fig. 3 of Ref. 27 with Fig. 2 first column, second raw panel). The slight discrepancy in the eigenvectors' directions points towards an overestimation, in our approach of the elastic constant stiffness of Ti-Ti bond along $b$, which is consistent with the $\mathrm{b}$ length overestimation in section IV.

This frequency at $175 \mathrm{~cm}^{-1}$ is close to the frequency of an anomalous mode observed in the Raman spectrum of Ref. 2 (named $\alpha$ ) which consists of a broad peak cen- tered around $160 \mathrm{~cm}^{-1}$ at room temperature. As the temperature decreases, the peak position shifts towards lower frequency values and its broadening reduces until it becomes indistiguishible from the Raman $\mathrm{A}_{g}$ mode allowed in the low symmetry phase. Due to its Lorentzian lineshape, this feature is attributed to low dimensional energy fluctuations of the spin system which manifest themselves in the form of quasielastic scattering of the Raman light and decrease as the temperature approaches the spin-Peierls transition point ${ }^{2}$. It is worth pointing out that this broad feature is detected when the Raman light is polarised along the $b$-axis (along which the spin chains lie) while it is totally absent when the light is polarised along the $a$-axis ${ }^{2}$.

Recently, the evolution with temperature of the Raman active $\mathrm{A}_{g}$ modes has been studied by Fausti et al 28 . At low temperature, along with the three unchanged HS $A_{g}$ phonons, they find nine additional modes as required by group theory analysis (see Table IV] upper pannel). Four of these nine modes persist above the spin-Peierls transition and become broader losing in intensity as the temperature increases. These modes are also visible in the Raman spectrum of Ref. 2 at low temperatures and the lowest frequency mode coincides with the feature $\alpha$ described above.

Combining the information of the Raman spectrum of Ref. 28 (where not only light polarisation $b b$ was used but also $b c$ ) with the information contained in Table IV] and in the phonon displacement eigenvectors (not shown), we are able to assign the experimental Raman frequencies to the calculated modes (Table $\mathrm{V}$ ). In the following reasoning we assume that the possible splitting of the LS modes' degeneracy due to quantum spin effects does not alter the picture qualitatively. We have already pointed out the fact that the $\mathrm{HS} \mathrm{B}_{3 g}$ modes remain almost identical to their LS (LS $A_{g}$ ) counterpart, as shown in Tables III and IV] At high temperature, the $\mathrm{B}_{3 q}$ modes are active with light polarisation $b c$ and therefore we expect their identical LS counterparts to have a similar activity. In the spectrum of Ref. 28, the two modes at 178.5 and 524.3 $\mathrm{cm}^{-1}$ are predominantly active with light polarisation $b c$, but very weakly active with polarisation $b b$ (this is confirmed in the spectrum of Ref. 2). We therefore assign these two modes to the two LS A $\mathrm{A}_{g}$ modes with displacement along $b$ at 140 and $431 \mathrm{~cm}^{-1}$. In order to assign the third mode of the same symmetry, we note that the modes at 296.5 and $305.3 \mathrm{~cm}^{-1}$ in the spectrum (with light polarisation $b b$ ) of Ref. 2 are weakly active. Assuming a systematic underestimation of the frequencies due to shortcomings of the theory (which will be discussed below) we assign the mode at $305.3 \mathrm{~cm}^{-1}$ to the calculated value at $289 \mathrm{~cm}^{-1}$. The remaining six LS A modes are then assigned straightforwardly in order of increasing value (see Table $\nabla$ for their assignment) and the three HS $\mathrm{A}_{g}$ modes remain unaltered at low temperature (compare Table $\mathrm{V}$ with Table III for their assignment).

By direct comparison, we find that while the three HS $\mathrm{A}_{g}$ modes are in good agreement with experiment, the 
TABLE V: Mode assignment for the twelve Raman active $\mathrm{A}_{g}$ modes listed in Table IV L.S. labels phonons that disappear at the SP transition $T_{c_{1}}$, I.P. those that survive up to the intermediate phase transition $\mathrm{T}_{c_{2}}$, H.S. represents the high symmetry phase phonons.

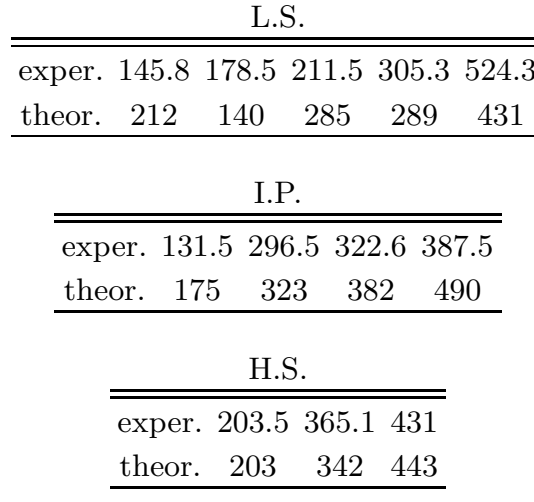

three LS $\mathrm{A}_{g}$ modes along $b$ ( $\mathrm{B}_{3 g}$ modes in the HS phase) are underestimated by $10-20 \%$ and the remaining six $\mathrm{A}_{g}$ modes of the LS phase are overestimated by 20-30\%. Possible origin of the above discrepancies could be: 1) the lack of a screened exchange term in the Hamiltonian which also causes an overestimation of $b ; 2$ ) the lack of spin-singlet dynamical correlations in the B3LYP Hamiltonian which usually renormalise the phononic spectrum and, as mentioned in section IV, may be responsible for the overestimation of the lattice parameter $b$.

Based on the above assignment, we are able to conclude that the mode at $175 \mathrm{~cm}^{-1}$ most probably evolves into the high temperature $\alpha$ feature of Ref. 2. The remaining five LS $\mathrm{A}_{g}$ modes show also dimerisation patterns which may be tentatively related to the broadening of their Raman spectra 2,28 due to spin fluctuations. Note, however, that two of the modes disappear at $\mathrm{T}_{c_{1}}$ due to a symmetry change in the intermediate phase. It would be interesting to perform a phonon calculation within the space group $13(P 2 / c)$, suggested in Ref. 28 as the intermediate phase symmetry, to countercheck the validity of our assignment as well as of the suggested space group.

\section{Role of pretransitional fluctuations and the spin-Peierls instability}

As seen above, the computed LS phonon calculations enable i) the comparison with Raman and infrared spectroscopy measurements performed for the zone center modes in the spin-Peierls phase as we showed in the previous section and ii) the prediction of the behavior of some zone boundary phonons in the HS phase as we will discuss in the following.

From our calculations, the $\alpha$ mode can be interpreted as a precursive mode characterised by a strong spinPeierls activity. In fact, this has a very broad lineshape and shows a large softening in a temperature range be- tween $\mathrm{T}_{c 1}$ and room temperature ${ }^{2}$. These features are associated with energy fluctuations of the magnetic and structural system. Concerning the latter, above the transition temperature $T_{c_{2}}$ strong incommensurate fluctuations have been detected in X-ray diffraction experiments ${ }^{27}$ and they are seen to persist up to room temperature. Our phonon calculation supports the lattice fluctuation scenario with effectively one-dimensional atom displacement paths, as shown in subsection VIB for the lowest frequency modes. In addition, anomalies in the electronic structure are also found in the ARPES spectra of the high temperature phase $\mathrm{e}^{\underline{\underline{6}}}$ which have been given little explanation so far.

Recently, the authors of Ref. 29 reported the observation of a zone boundary acoustic phonon in $\mathrm{TiOCl}$ which softens at $\mathrm{T}_{c_{2}}$ 30,31. They identify this mode as the one that drives the spin-Peierls transition and estimate its bare phonon frequency to be $\Omega_{0} \sim 217 \mathrm{~cm}^{-1}$. In our calculation of the relevant zone boundary modes in the HS for the unit cell doubling along $b$ (section VI A) we found that the doubly degenerate mode $\left(A_{g}, B_{u}\right)$ at 175 $\mathrm{cm}^{-1}$ shows features of a spin-Peierls phonon in its vibrational pattern (see Fig. 2). While we cannot say at this point whether this corresponds to an acoustic or to an optical zone boundary phonon of the HS phase, its energy is close to the bare phonon energy estimated by Abel et al $\stackrel{29}{\underline{*}}$. Moreover, one could then speculate that there is a direct relation between the $\alpha$ phonon observed in Raman spectroscopy with the interpreted spin-Peierls phonon observed in inelastic X-ray experiments.

A last remark concerns the incommensurate region between $T_{c_{2}}$ and $T_{c_{1}}$. This region shows incommensurate wave vectors shifted from the commensurate wave vector $\left(0, \frac{1}{2}, 0\right)$ by the amount $( \pm \Delta H, \pm \Delta K, 0)$. It has been argued that the origin of this incommensurability originates from competing arrangements of bond patterns in TiOCl ${ }^{5,13,27}$ and more recently in terms of a model of discommensurations separating commensurate from dimerized regions 29 . The existence of degenerate modes enforces the argument of competing patterns in the incommensurate region with possible coexisting domains.

\section{CONCLUSION}

In summary, we have presented within hybrid density functional theory a complete description of the phonon modes (Raman and infrared active) in $\mathrm{TiOCl}$ both in the space group Pmmn (high symmetry phase) and in the space group $P 2_{1} / m$ which is the symmetry of the spinPeierls phase. Comparison with experiment and with results obtained from other functionals shows that nonlocal correlations as implemented in the hybrid B3LYP approach are important in order to obtain an improved description of the modes. This study supports the correlated nature of $\mathrm{TiOCl}$.

Our analysis of the zone center high-symmetry modes (space group Pmmn) and some relevant zone boundary 
high-symmetry modes (space group $P 2_{1} / m$ ) allows us to identify the anomalous $\alpha$ mode observed in Raman scattering as a probable precursor of the spin-Peierls mode strongly coupled to the spin system. Moreover, the triangular geometry of bonds between Ti atoms belonging to neighboring chains running along $b$ leads to the existence of six $\mathrm{A}_{g}$ zone boundary modes degenerate with six $\mathrm{B}_{u}$ zone boundary modes (for the undistorted system), a phenomenon which has not been observed in other known spin-Peierls systems and which contributes to the anomalous behavior of $\mathrm{TiOCl}$.

Some of the low symmetry modes show effectively onedimensional atomic displacement paths supporting the important role of lattice fluctuations in addition to the magnetic ones

\section{Acknowledgments}

We would like to thank R. Claessen, C. Gros, M. Grüninger, H.O. Jeschke, P. Lemmens, T. SahaDasgupta, M. Sing , A. Dobry, M. Mostovoy, D. Khomskii and G. Mallia for useful discussions. R.V. thanks the Deutsche Forschungsgemeinschaft for financial support through FOR412 and SFB/TRR49 grants and the NSF through Grant. No. PHY05-51164.
1 A. Seidel, C. A. Marianetti, F. C. Chou, G. Ceder, and P. A. Lee, Phys. Rev. B 67, 020405 (2003).

2 P. Lemmens, K. Y. Choi, G. Caimi, L. Degiorgi, N. N. Kovaleva, A. Seidel, and F. C. Chou, Phys. Rev. B 70, 134429 (2004).

3 T. Saha-Dasgupta, R. Valentí, H. Rosner, and C. Gros, Europhys. Lett. 67, 63 (2004).

${ }^{4}$ P. Lemmens, K. Y. Choi, R. Valentí, T. Saha-Dasgupta, E. Abel, Y. S. Lee, and F. C. Chou, New J. Phys. 2, 74 (2005).

5 R. Rückamp, J. Baier, M. Kriener, M. W. Haverkort, T. Lorenz, G. S. Uhrig, L. Jongen, A. Möller, G. Meyer, and M. Grüninger, Phys. Rev. Lett. 95, 097203 (2005).

6 M. Hoinkis, M. Sing, J. Schäfer, M. Klemm, S. Horn, H. Benthien, E. Jeckelmann, T. Saha-Dasgupta, L. Pisani, R. Valentí, et al., Phys. Rev. B 72, 125127 (2005).

7 L. Pisani and R. Valentí, Phys. Rev. B 71, 180409R (2005).

8 M. Shaz, S. van Smaalen, L. Palatinus, M. Hoinkis, M. Klemm, S. Horn, and R. Claessen, Phys. Rev. B 71, 100405 (2005).

9 J. Hemberger, M. Hoinkis, M. Klemm, M. Sing, R. Claessen, S. Horn, and A. Loidl, Phys. Rev. B 72, 012420 (2005).

10 T. Saha-Dasgupta, A. Lichtenstein, and R. Valentí, Phys. Rev. B 71, 153108 (2005).

11 E. M.-H. L. Craco, M.S. Laad, J. Phys.: Condens. Matter 18, 10943 (2006).

12 T. Saha-Dasgupta, A. Lichtenstein, M. Hoinkis, S. Glawion, M. Sing, R. Claessen, and R. Valentí, condmat/0612166 (2006).

13 J. P. Clancy, B. D. Gaulin, K. C. Rule, J. P. Castellan, and F. C. Chou, Phys. Rev. B 75, 100401 (2007).

14 M. Hoinkis, M. Sing, S. Glawion, L. Pisani, R. Valenti, S. van Smaalen, M. Klemm, S. Horn, and R. Claessen, Phys. Rev. B 75, 245124 (2007).
15 L. Palatinus, A. Schoenleber, and S. van Smaalen, Acta Cryst. C 61, i48 (2005).

16 A. D. Becke, J. Chem. Phys. 98, 5648 (1993).

17 P. J. Stephens, F. J. Devlin, C. F. Chabalowski, and M. J. Frisch, J. Phys. Chem. 98, 11623 (1994).

18 V. R. Saunders, R. Dovesi, C. Roetti, R. Orlando, C. M. Zicovich-Wilson, N. M. Harrison, K. Doll, B. Civalleri, I. J. Bush, P. D'Arco, et al., CRYSTAL06 User's Manual, Università di Torino (Torino, 2006).

19 H. J. Monkhorst and J. D. Pack, Phys. Rev. B 13, 5188 (1976).

20 H. B. Schlegel, J. Comp. Chem. 3, 214 (1982).

21 T. Imai and F. Chou, cond-mat/0301425 (????).

${ }^{22}$ X.-B. Feng and N. M. Harrison, Phys. Rev. B 69, 035114 (2004).

23 J. P. Perdew, K. Burke, and M. Ernzerhof, Phys. Rev. Lett. 77, 3865 (1996).

24 C. Lee, W. Yang, and R. G. Parr, Phys. Rev. B 37, 785 (1988).

25 G. Caimi, L. Degiorgi, N. N. Kovaleva, P. Lemmens, and F. C. Chou, Phys. Rev. B 69, 125108 (2004).

26 M. Grueninger, priv. comm. (2007).

27 A. Schonleber, S. van Smaalen, and L. Palatinus, Phys. Rev. B 73, 214410 (2006).

28 D. Fausti, T. T. A. Lummen, C. Angelescu, R. Macovez, J. Luzon, R. Broer, P. Rudolf, P. H. M. van Loosdrecht, N. Tristan, B. Buchner, et al., Phys. Rev. B 75, 245114 (2007).

29 E. T. Abel, K. Matan, F. C. Chou, E. D. Isaacs, D. E. Moncton, H. Sinn, A. Alats, and Y. S. Lee, arXiv:0708.0717 (2007).

30 C. Gros and R. Werner, Phys. Rev. B 58, 14677 (1998).

31 A. Dobry, D. C. Cabra, and G. L. Rossini, Phys. Rev. B 75, 045122 (2007). 